\title{
Política, elecciones y democracia en Costa Rica (1889-1914). Una nota historiográfica
}

\section{Iván Molina Jiménez ${ }^{1}$}

Recepción: 9 de julio de 2015/ Aprobación: 28 de agosto de 2015

\section{Resumen}

El objetivo principal de este artículo es analizar la producción historiográfica que estudia la política electoral costarricense de los años 1889-1914. Dicha escogencia se explica por dos razones: se trata de uno de los períodos más estudiados por los investigadores y constituye, a la vez, una de las etapas fundamentales de la formación de la democracia en Costa Rica. El análisis efectuado demuestra que las investigaciones realizadas, inicialmente descriptivas y tradicionales, tendieron a volverse más sofisticadas a partir de la década de 1980. Sin embargo, fue sólo a partir del decenio de 1990 que los trabajos académicos empezaron a considerar los aspectos institucionales en marcos más amplios y a introducir una indispensable perspectiva comparativa.

\section{Palabras clave}

Historiografía; política; elecciones; democracia; Costa Rica

\section{Abstract}

The main objective of this paper is to study the historiography of the Costa Rican electoral politics developed between 1889 and 1914. The choice for this period is explained by two reasons: it is one of the most studied periods and is one of the key phases in the formation of democracy in Costa Rica. The analysis shows that research was initially descriptive and traditional, and then since the 1980s tended to become more sophisticated, however, it was from the 1990s that scholars began to consider the institutional aspects in broader frameworks and started to introduce a necessary and broader comparative perspective.

\section{Keywords}

Historiography; politcs; elections; democracy; Costa Rica

1 Costarricense. Máster en Historia por la Universidad de Costa Rica (UCR). Catedrático de la Escuela de Historia e investigador del Centro de Investigación en Identidad y Cultura Latinoamericanas (CIICLA), ambas de la UCR. Correo electrónico: IVAN.MOLINA@ucr.ac.cr 


\section{Resumo}

O principal objetivo deste artigo é analisar a historiografia sobre a política eleitoral da Costa Rica dos anos 1889-1914. Esta escolha é explicada por dois motivos: é um dos períodos mais estudados por pesquisadores e é, ao mesmo tempo, um dos principais etapas na formação da democracia na Costa Rica. A análise mostra que a pesquisa inicialmente descritivo e tradicional, tendem a se tornar mais sofisticado desde os anos 1980. A análise mostra que a pesquisa inicialmente descritivo e tradicional, tendem a se tornar mais sofisticado desde os anos 1970.

No entanto, foi somente a partir da década de 1990 que o trabalho acadêmico começou a considerar os aspectos institucionais em um contexto mais amplo e de introduzir uma perspectiva comparativa essencial.

\section{Palabras chave}

Historiografia; política; eleições; democracia; Costa Rica

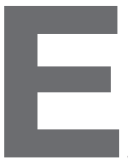

I propósito de este artículo es hacer un balance de la producción historiográfica que ha analizado la política electoral costarricense de los años 1889-1914, uno de los períodos más investigados por los historiadores y otros especialistas en Ciencias Sociales precisamente porque constituyó una etapa fundamental en la formación de la democracia costarricense. Para cumplir con el objetivo indicado, el trabajo ha sido dividido en tres secciones principales: en la primera, se consideran las contribuciones realizadas hasta comienzos de la década de 1970, cuando el proceso de renovación de la historiografía costarricense apenas empezaba; en la segunda, se examinan los aportes dados a conocer en la década de 1980, en particular los de Orlando Salazar Mora; y en la tercera, se exploran las principales innovaciones introducidas entre finales del siglo XX e inicios del siglo XXI.

\section{Primeros aportes}

Hasta 1950, el conocimiento de la política electoral costarricense de los años 1889-1914 se basaba básicamente en cinco contribuciones principales: la Cartilla histórica de Costa Rica, de Ricardo Fernández Guardia (1909); la biografía de Rafael Iglesias Castro, elaborada por Hernán G. Peralta (1928); el estudio sobre el obispo Bernardo Augusto Thiel, preparado por Víctor Manuel Sanabria Martínez (1941); la crónica de los comicios de 1889 y del levantamiento popular posterior, escrita por José María Pinaud (1942); y la síntesis de Harold H. Bonilla sobre las figuras presidenciales (1942). De estos trabajos, el más detallado fue el de Sanabria, aunque todas esas obras reconocieron el carácter estratégico del período antes referido, en el que se profundizó la secularización social -intensificada a partir de las reformas liberales- se expandió la 
cultura impresa, empezó a consolidarse una esfera pública y se constituyó un sistema estable de partidos políticos.

Ciertamente, entre 1933 y 1936 el ex presidente Cleto González Víquez publicó en la Revista Jurisprudencia un extenso estudio sobre el sufragio, en el que analizó la legislación electoral y las luchas por el poder; sin embargo, esta valiosa contribución se concentró en el período 1824-1871, prestó más atención a los procesos políticos que a las elecciones, no siempre consignó los resultados de las votaciones y, cuando lo hizo, con frecuencia los indicó apenas de manera general. Además, no consideró debidamente la importancia de cambios institucionales fundamentales como los introducidos en la Constitución de 1848, que restringieron la ciudadanía, y los incorporados en la de 1859, que prácticamente establecieron el sufragio universal masculino. Posteriormente estos materiales fueron recopilados en un libro, que incluyó un capítulo inédito (González Víquez, 1958, pp. 15-472).

Entre 1950 y 1972, la política costarricense de finales del siglo XIX e inicios del XX se conoció mejor gracias al importante libro de Rafael Obregón Loría sobre los conflictos políticos y militares, publicado en 1951, del cual circuló una edición ampliada y actualizada tres décadas después (Obregón Loría, 1951 y 1981); y a diversas tesis de licenciatura presentadas en la Universidad de Costa Rica y a algunos estudios independientes que tendieron a privilegiar la investigación de figuras políticas y de administraciones presidenciales (Hernández Faerrón, 1955; García Zúñiga, 1956; Quirós Berrocal, 1957; Alonso Quesada, 1961; Montiel Héctor, 1970; Martínez Arias, 1971; Brenes Rosales, 1972; Rodríguez Vega, 1971; Pacheco León, 1972). Frente a esta tendencia más tradicional, una decisiva ruptura teórica y metodológica ocurrió a finales de la década de 1960, al presentar Carlos Araya Pochet una disertación sobre el Partido Liberación Nacional, fundado en 1951, con un enfoque más analítico (1968). De 1970 en adelante, esta significativa innovación historiográfica se extendió al período 1889-1914, cuando Clara María di Luca Laurito (1973), Orlando Salazar Mora (1973), Carlos Calvo Gamboa (1974) y Germán Solís Barquero (1974) dieron a conocer tesis sobre el Partido Unión Católica, el Partido Republicano y el papel jugado por su líder, Máximo Fernández Alvarado, el Partido Civil de Rafael Iglesias Castro y la participación política de Carlos Durán Cartín; además, Carlos Morales Castro (1971) investigó la relación entre prensa y política a finales del siglo XIX, y Carlos Ramírez Mata analizó la influencia del presidente Ricardo Jiménez Oreamuno en el sistema político costarricense (1973) .

También en la década de 1970 circularon dos obras que ampliaron el conocimiento sobre política electoral del período aquí considerado: Nelson Chacón Pacheco publicó un detallado estudio acerca de la legislación costarricense relacionada con los comicios, en el que consideró las últimas décadas del siglo XIX y primeras del XX (1975, pp. 130-169); y Samuel Stone realizó el primer análisis a fondo del sistema electoral de esa época, en el que examinó -aunque brevemente- aspectos fundamentales como las reformas 
electorales, el surgimiento de los partidos políticos, la creciente asistencia de la población a las urnas, los resultados de las votaciones y el papel jugado por los gamonales (1975, pp. 222-224, 233-235, 268-270).

\section{Nuevas perspectivas}

De los investigadores referidos, el único que se especializó en el estudio del período 1889-1914 fue Salazar Mora: en 1980, presentó en Francia una tesis de doctorado en la que profundizó en el análisis del sistema electoral costarricense; más tarde, elaboró algunos valiosos trabajos sobre temas específicos (la Comisión Permanente y las leyes electorales de 1889, 1893 y 1913), y culminó sus esfuerzos con una síntesis fundamental, publicada en 1990. En este libro, que inicia en 1870, consideró la vida política, la organización jurídicoadministrativa del Estado, los procesos de acceso al poder y de constitución de partidos políticos, las elecciones presidenciales y la legitimación de lo que él denominó la "democracia liberal” (Salazar Mora, 1980, 1981, 1986, 1990).

Para producir esa síntesis, Salazar Mora no solo recuperó problemáticas básicas provenientes de distintas corrientes teóricas (Pierre Bourdieu, Maurice Duverger, Gino Germani, Hugues Portelli y Nicos Poulantzas), sino que, al reconsiderar sus propios aportes, incorporó las nuevas contribuciones producidas por otros investigadores sociales, en particular las de José Luis Vega Carballo acerca de la formación del Estado costarricense (1981). Además, integró los hallazgos de una nueva generación de historiadores, especialmente los de Ástrid Fischel Volio acerca de la reforma educativa de 1886 (1986), la de Patricia Badilla Gómez sobre la reforma jurídica de la década de 1880 (1988), la de Mercedes Muñoz Guillén que analizó la abolición del ejército (1988) y la de Claudio Vargas Arias que investigó el conflicto entre el Estado y la Iglesia católica (1989). No obstante su exhaustividad, el libro dejó de lado un importante aporte: el análisis de Ólger González Murillo sobre el desarrollo del sector público, en particular el de las carteras de Guerra y de Instrucción Pública (1980).

Casi un cuarto de siglo después de su publicación, la síntesis de Salazar Mora se mantiene como una obra de consulta indispensable; sin embargo, las nuevas vías de investigación que posibilitó, pronto condujeron a resultados que superaron algunos de sus planteamientos. Steven Palmer demostró que, lejos de corresponderse con el modelo "laissez-fairiano", el Estado liberal costarricense, desde muy temprano, impulsó activas políticas sociales y culturales, que fueron decisivas para la construcción cultural de la nación (1999, pp. 99-117; 2004, pp. 257-323). Igualmente, Fabrice E. Lehoucq consideró la problemática del origen de la democracia en Costa Rica desde una estratégica perspectiva comparativa (Lehoucq, 1991). Ambos investigadores, además, procuraron integrar en sus interpretaciones los resultados de las nuevas investigaciones realizadas en los campos de la historia económica, social y cultural, 
un interés ya presente en un trabajo pionero de Mario Samper Kutschbach publicado en 1989 (pp. 164-167 y 173-174).

\section{Contribuciones recientes}

En el bienio 1996-1997, Hugo Vargas González y Erick Ovares Rodríguez profundizaron en el análisis de la política y los procesos electorales ya investigados por Salazar Mora. De la contribución del primero debe destacarse que, dado que partió de 1821, pudo efectuar un valioso estudio de la formación del sistema político-electoral, lo que le permitió destacar no solo que fue con la Constitución de 1859 que empezó a consolidarse el sufragio universal masculino en el país, sino prestarle la atención debida a la elección presidencial de 1886 -a la que Salazar Mora no le dio importancia-, la primera en la que se volvieron a organizar partidos políticos desde las iniciativas similares emprendidas en la década de 1860 (Vargas González, 1996). A su vez, el segundo concentró su esfuerzo en elaborar un detallado e imaginativo análisis de la transacción de 1901, que posibilitó el retiro del poder de Iglesias Castro; curiosamente, este autor no consultó la importante síntesis publicada por Salazar Mora en 1990 (Ovares Rodríguez, 1997).

Igualmente, mayores detalles relacionados con los comicios y con los cambios en la legislación electoral realizados entre 1885 y 1914 fueron aportados por Clotilde Obregón Quesada en un libro publicado en el año 2000 (pp. 194257.). En ese mismo año, circuló un estudio de Patricia Fumero Vargas que analizó la elección presidencial de 1913 (la primera en la que se puso en práctica el voto directo) desde la perspectiva de la fiesta electoral. Con base en este enfoque, la autora detalló las diversas actividades que realizaban los partidos para atraer a sus potenciales votantes, tanto en las áreas urbanas como en las rurales, y documentó la amplia participación que tenían las mujeres, pese a que carecían del derecho al voto (Fumero Vargas, 2000, pp. 41-57). Sería interesante en futuros estudios investigar la relación entre la fiesta electoral y la fiesta de la independencia (Díaz Arias, 2007).

Trabajos dados a conocer entre finales del siglo XX y comienzos del XXI empezaron a tratar -con una perspectiva comparativa- algunos temas ampliamente mencionados en los estudios existentes, pero no investigados de manera sistemática, como el fraude electoral, la asistencia a las urnas y la composición de los electores de segundo grado. A diferencia de la síntesis de Salazar Mora, estas nuevas contribuciones mostraron que la política electoral tuvo una base decisivamente popular, que el abstencionismo se redujo de manera significativa en la década de 1900, cuando casi el cien por ciento de los costarricenses adultos estaban empadronados para votar; que los partidos -y sus representantes en el Congreso- desarrollaron mecanismos para controlar las prácticas fraudulentas; y que entre 1885 y 1914 las demandas del electorado contribuyeron a que las políticas públicas se orientaran a la satisfacción de 
necesidades de las comunidades urbanas y rurales, sobre todo en las áreas de educación, salud e infraestructura (Molina Jiménez, 2005; Molina Jiménez y Lehoucq, 1999 y 2002; Céspedes Brenes, Hernández Abarca y Muñoz Zeledón, 2002; Rojas Sandoval, 2002, pp. 111-120).

Con base en lo aportado por quienes lo precedieron, Esteban Sánchez Solano recién dio a conocer el estudio más detallado sobre un partido político costarricense fundado en el siglo XIX: el Unión Católica. Al revisitar este tema, y considerar de manera amplia aspectos como su proceso de formación, sus organizaciones de base (en particular los círculos y los clubes católicos), las estrategias que utilizó para movilizar el voto, las características de sus dirigentes civiles y eclesiásticos, su imaginario político y su inserción en la competencia electoral, el autor hizo una contribución fundamental para comprender el ciclo político iniciado con las elecciones de 1885 y que finalizó con la transacción de 1901 (Sánchez Solano, 2013).

Pese a estos aportes, y a la exhaustiva revisión documental efectuada, el estudio de Sánchez Solano evidencia un dominio limitado de las problemáticas y metodologías propiamente politológicas y un marco comparativo insuficiente -circunscrito a una sección teórica introductoria- que no profundiza en el análisis de las especificidades costarricenses en relación con América Latina; tampoco logró ubicar de forma adecuada la experiencia investigada en el contexto de la primera ola internacional de democratización (Tovar, 2009; Tilly, 2007; Przeworski, 2008) (de hecho, la cuestión de la democracia es sistemáticamente eludida). Por último, la valiosa producción académica reciente, publicada en inglés sobre los partidos políticos del siglo XIX, está casi ausente en su trabajo.

\section{Conclusión}

A lo largo de este análisis, quedó claro que al investigar el período 18891914 se pasó de estudios que privilegiaban la descripción de los hechos políticos, a otros que empezaron a plantear la investigación de la política electoral en términos de procesos y tendencias. De igual forma, se transitó de enfoques dominados por perspectivas estructuralistas, que no prestaban la debida atención a los contextos económicos, sociales y culturales, a estrategias metodológicas que procuraron analizar lo institucional en marcos más amplios. Por último, algunas de las contribuciones más recientes incorporaron también perspectivas comparativas, aunque no siempre plenamente desarrolladas.

En 1990, Salazar Mora terminó su libro con un fuerte acento en el carácter limitado y restrictivo de la democracia liberal, a la que conceptuaba como de tipo "oligárquico"; una década más tarde, las nuevas investigaciones empezaron a destacar que fue justo en el período antes referido que Costa Rica transitó decisivamente hacia la democracia, en el marco de la primera oleada internacional de democratización. En efecto, entre 1885 y 1914, se constituyeron en 
el país prácticas electorales estables y periódicas, con espacios institucionales cada vez más amplios para los grupos de oposición, dos tendencias que se combinaron de manera estratégica con la expansión del sufragio, la canalización de las demandas populares por medio de los partidos y el ascenso de políticas públicas socialmente orientadas.

Innovador en su momento, el libro que publicó Salazar Mora en 1990, aun con las limitaciones señaladas, conserva su importancia como una contribución básica al conocimiento del sistema político, de la formación del Estado y de la política electoral del período 1885-1914. Desafortunadamente, dicha síntesis no fue debidamente debatida en la última década del siglo XX. De las tres reseñas que originó, una se limitó apenas a describir el contenido de la obra (Fonseca Corrales, 1991), otra la descalificó por razones sobre todo ideológicas (Quesada Monge, 1991), y solo la tercera procuró dialogar de manera crítica con los planteamientos del autor (Molina Jiménez, 1994).

Un cuarto de siglo después, los nuevos aportes realizados posibilitan un enfoque más amplio, complejo y sofisticado sobre el proceso de formación de la democracia y el papel jugado por distintas fuerzas políticas, y acerca de la relación entre los factores institucionales y los económicos, sociales y culturales. Además, está en marcha ya una tendencia que considera el caso de Costa Rica a partir de una decisiva perspectiva comparativa, que permite ubicar al país en el marco de la primera oleada internacional de democratización. Cierto es que el conocimiento de tales contribuciones no se ha extendido suficientemente, como lo demuestran la importante tesis de Sánchez Solano y un libro reciente de Álvarez Garro (2011, pp. 25-69), el cual, al investigar el "mito democrático" costarricense, ni siquiera consultó el libro de Salazar Mora de 1990. Pese a estos vacíos y omisiones, los avances logrados en los últimos cinco lustros ofrecen desafiantes puntos de partida para investigar de manera comparada el origen de la especificidad democrática costarricense.

\section{Referencias}

Alonso Quesada, Elena Isabel. (1961). Antecedentes y primer gobierno de Ricardo Jiménez (Tesis de Licenciatura en Historia). Universidad de Costa Rica, San José, Costa Rica.

Álvarez Garro, Laura. (2011). El mito democrático costarricense. La constitución de la práctica política en períodos de conflicto social. México: Facultad Latinoamericana de Ciencias Sociales.

Araya Pochet, Carlos. (1968). Historia de los partidos políticos: Liberación Nacional (Tesis de Licenciatura en Historia). Universidad de Costa Rica, San José, Costa Rica. 
Badilla Gómez, Patricia. (1988). Estado, ideología y derecho: la reforma jurídica costarricense: 1882-1888 (Tesis de Maestría en Historia). Universidad de Costa Rica, San José, Costa Rica.

Bonilla, Harold H. (1942). Nuestros presidentes. San José: Soley \& Valverde.

Brenes Rosales, Raymundo. (1972). Don José Joaquín Rodríguez, un dictador constitucional (Tesis de Licenciatura en Historia). Universidad de Costa Rica, San José, Costa Rica.

Calvo Gamboa, Carlos. (1974). Rafael Iglesias y su partido civilista (Tesis de Licenciatura en Historia). Universidad Nacional, Heredia, Costa Rica.

Céspedes Brenes, Adriana María, Hernández Abarca, Daisy \& Muñoz Zeledón, Sylvia María. (2002). Hasta los muertos votan: el fraude en los procesos electorales municipales de Costa Rica 1901-1948 (Memoria de Licenciatura en Historia). Universidad de Costa Rica, San José, Costa Rica.

Chacón Pacheco, Nelson. (1975). Nuestras leyes electorales. San José: Editor no identificado.

Díaz Arias, David. (2007). La fiesta de la independencia en Costa Rica, 18211921. San José: Editorial de la Universidad de Costa Rica.

Fernández Guardia, Ricardo. (1909). Cartilla histórica de Costa Rica. San José: Alsina.

Fischel Volio, Ástrid. (1986). Educación y consenso: la reforma educativa en el desarrollo socio-político costarricense. 1885-1889 (Tesis de Maestría en Historia). Universidad de Costa Rica, San José, Costa Rica.

Fonseca Corrales, Elizabeth. (1991). Orlando Salazar Mora. 1990. El apogeo de la república liberal en Costa Rica. 1870-1914. San José: Editorial Universidad de Costa Rica.

Fumero Vargas, Patricia. (2000). Cultura política y fiesta electoral en Costa Rica a inicios del siglo XX. Revista de Ciencias Sociales (89), 41-57.

García Zúñiga, Didier. (1956). Don Ascensión Esquivel: su personalidad y su labor de estadista (Tesis de Licenciatura en Historia). Universidad de Costa Rica, San José, Costa Rica.

González Murillo, Ólger. (1980). Análisis histórico de la evolución burocrática en las distintas carteras gubernamentales de Costa Rica con énfasis en los sectores de Instrucción - Pública y Guerra (militar): 1871-1919 (Tesis de Licenciatura en Historia). Universidad de Costa Rica, San José, Costa Rica. 
González Víquez, Cleto. (1958). El sufragio en Costa Rica ante la historia y la legislación. En Cleto González Víquez, Obras históricas, t. I. San José: Editorial Universitaria, 15-472.

Hernández Faerrón de Jaén, Minerva. (1955). Don Rafael Iglesias Castro (Tesis de Licenciatura en Historia). Universidad de Costa Rica, San José, Costa Rica.

Lehoucq, Fabrice. (1991). The Origins of Democracy in Costa Rica in Comparative Perspective (Tesis de Doctorado en Ciencias Políticas). Duke University, Durham, Estados Unidos.

Di Luca Laurito, Clara María. (1973). Partido Unión Católica: primer partido ideológico de Costa Rica (Tesis de Licenciatura en Historia). Universidad de Costa Rica, San José, Costa Rica.

Martínez Arias, Enrique. (1971). Cleto González Víquez en la primera década del siglo XX (Tesis de Licenciatura en Historia). Universidad de Costa Rica, San José, Costa Rica.

Molina Jiménez, Iván \& Lehoucq, Fabrice. (1999). Urnas de lo inesperado. Fraude electoral y lucha política en Costa Rica (1901-1948). San José: Editorial de la Universidad de Costa Rica.

Molina Jiménez, Iván \& Lehoucq, Fabrice. (2002). Stuffing the Ballot Box. Fraud, Electoral Reform, and Democratization in Costa Rica. Cambridge: Cambridge University Press.

Molina Jiménez, Iván. (1994). El apogeo de la República Liberal en Costa Rica (1870-1914), de Orlando Salazar. Rumbo (481) (1 de marzo), 39-40.

Molina Jiménez, Iván. (2005). Demoperfectocracia. La democracia pre-reformada en Costa Rica (1885-1948). Heredia: Editorial Universidad Nacional.

Montiel Héctor, Aida María. (1970). Historia del Poder Ejecutivo en Costa Rica: 1902-1924 (Tesis de Licenciatura en Historia). Universidad de Costa Rica, San José, Costa Rica.

Morales Castro, Carlos. (1971). Diarios costarricenses nacen y mueren a la sombra de la política: una época en la historia del diarismo (Tesis de Licenciatura en Historia). Universidad de Costa Rica, San José, Costa Rica.

Muñoz Guillén, Mercedes. (1989). El Estado y la abolición del ejército. 19141949 (Tesis de Maestría en Historia). Universidad de Costa Rica, San José, Costa Rica.

Obregón Loría, Rafael. (1951). Conflictos militares y políticos de Costa Rica. San José, Imprenta La Nación. 
Obregón Loría, Rafael. (1981). Hechos militares y políticos. Alajuela: Museo Histórico Cultural Juan Santamaría.

Obregón Quesada, Clotilde. (2000). El proceso electoral y el Poder Ejecutivo en Costa Rica. San José: Editorial Universidad de Costa Rica, 2000.

Ovares Rodríguez, Erik. (1997). Práctica política en Costa Rica: 1889-1924. Cooptación, penetración ideológica y cultura política (Tesis de Licenciatura en Historia). Universidad Nacional, Heredia, Costa Rica.

Pacheco, León. (1972). Máximo Fernández. San José: Ministerio de Cultura, Juventud y Deportes.

Palmer, Steven. (1999). Adiós laissez-faire: la política social en Costa Rica. 1880-1940. Revista de Historia de América (124), 99-117.

Palmer, Steven. (2004). "Sociedad anónima, cultura oficial: inventando la nación en Costa Rica, 1848-1900”. Iván Molina Jiménez \& Steven Palmer (eds.), Héroes al gusto y libros de moda. Sociedad y cambio cultural en Costa Rica (1750-1900), 2da. edición. San José: Editorial Universidad Estatal a Distancia, 257-323.

Peralta, Hernán G. (1928). Don Rafael Yglesias: apuntes para su biografía. San José: Imprenta y Librería Trejos.

Pinaud, José María. (1942). El 7 de noviembre de 1889: la epopeya del civismo costarricense. San José: Imprenta La Tribuna.

Przeworski, Adam. (2009). Conquered or Granted? A History of Suffrage Extensions. British Journal of Political Science, 39(2), 291-321.

Quesada Monge, Rodrigo. (1991). Positivismo liberal y liberalismo positivista. A propósito de un libro de Orlando Salazar Mora. Revista de Historia (23), 251-256.

Quirós Berrocal, Ana Cecilia. (1957). Don Ascensión Esquivel Ibarra: adalid de la libertad dentro del orden (Tesis de Licenciatura en Historia). Universidad de Costa Rica, San José, Costa Rica.

Ramírez Mata, Carlos. (1973). Ricardo Jiménez y su influencia en el sistema político costarricense (Tesis de Licenciatura en Historia). Universidad de Costa Rica, San José, Costa Rica.

Rodríguez Vega, Eugenio. (1971). Los días de don Ricardo. San José: Editorial Costa Rica.

Rojas Sandoval, Francisco Javier. (2002). La cultura política de las clases trabajadoras urbanas de Costa Rica: el caso de los carpinteros y ebanistas (1890-1943). Revista de Historia (46), 111-148. 
Salazar Mora, Orlando. (1973). Máximo Fernández y el Partido Republicano (Tesis de Licenciatura en Historia). Universidad de Costa Rica, San José, Costa Rica.

Salazar Mora, Orlando. (1980). Le système politique au Costa Rica: 1889-1919 (Tesis de Doctorado en Historia). Universidad de París III, París, Francia.

Salazar Mora, Orlando. (1981). La Comisión Permanente y la suspensión del orden constitucional. Revista de Ciencias Jurídicas (44), 19-48.

Salazar Mora, Orlando. (1986). El sistema electoral costarricense: un análisis del período 1889-1919. Avances de Investigación del Centro de Investigaciones Históricas (20), 1-23.

Salazar Mora, Orlando. (1990). El apogeo de la república liberal en Costa Rica (1870-1914). San José: Editorial de la Universidad de Costa Rica.

Samper Kutschbach, Mario. (1989). Fuerzas sociopolíticas y procesos electorales en Costa Rica. Revista de Historia (No. especial), 157-222.

Sanabria Martínez, Víctor Manuel. (1941). Bernardo Augusto Thiel, segundo obispo de Costa Rica: apuntes históricos. San José: Lehmann.

Sánchez Solano, Esteban. (2013). La participación político-partidista de la lglesia: el Partido Unión Católica y sus estrategias de movilización política en el marco del conflicto entre la Iglesia católica y el Estado liberal en Costa Rica (1889-1898) (Tesis de Maestría en Historia). Universidad de Costa Rica, San José, Costa Rica.

Solís Barquero, Germán. (1974). El Dr. Carlos Durán C., su participación en la política costarricense (Tesis de Licenciatura en Historia). Universidad de Costa Rica, San José, Costa Rica.

Stone, Samuel. (1975). La dinastía de los conquistadores. La crisis del poder en la Costa Rica contemporánea. San José: Editorial Universitaria Centroamericana.

Tilly, Charles. (2007). Democracy. Cambridge: Cambridge University Press.

Tovar, Jesús. (2009). Las primeras democracias en Hispanoamérica. Política y Gobierno, XVI(1), 71-113.

Vargas Arias, Claudio. (1989). Liberalismo y la consolidación del Estado en Costa Rica: el encuentro entre el Estado liberal y la Iglesia Católica, 18801895 (Tesis de Maestría en Historia). Universidad de Costa Rica, San José, Costa Rica.

Vargas González, Hugo. (1996). Procesos electorales y luchas de poder en Costa Rica. Estudio sobre el origen del sistema de partidos (1821-1902) 
(Tesis de Licenciatura en Historia). Universidad de Costa Rica, San José, Costa Rica.

Vega Carballo, José Luis. (1981). La formación del Estado nacional en Costa Rica. San José: Instituto Centroamericano de Administración Pública. 